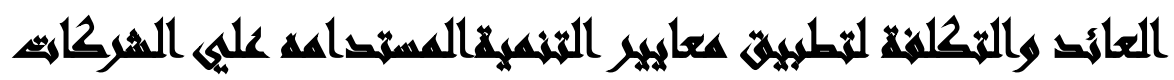

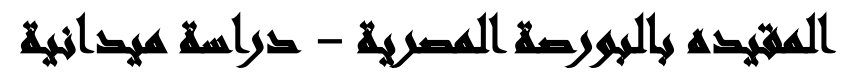

[IT]

خالد حسين أحمد أبو حسين(')- محمد أحمد لطفي محمد وهدان(') نجلاء أحمد فراج علي ( ) كلية تجارة، جامعة عين شمس

\section{المُسخلفه}

تهدف هذه الدراسة الى البحث فى بيان تأثير اتباع الثركات المسجله فى البورصه

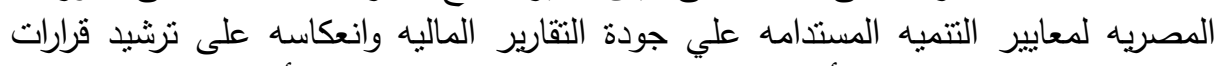

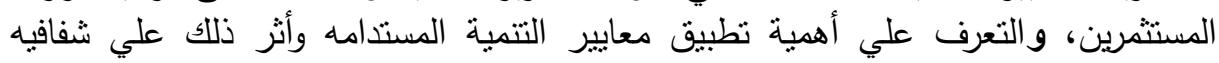

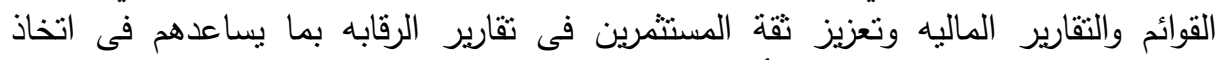

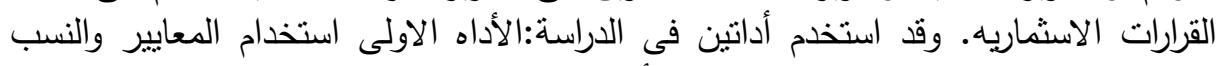

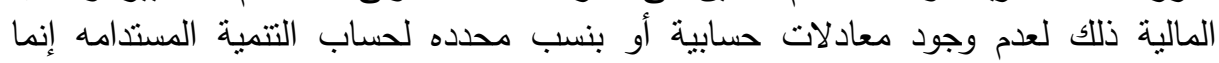

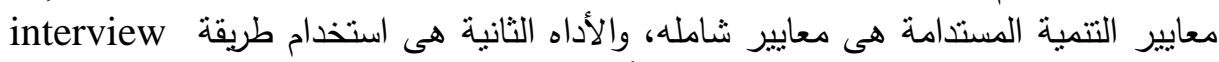

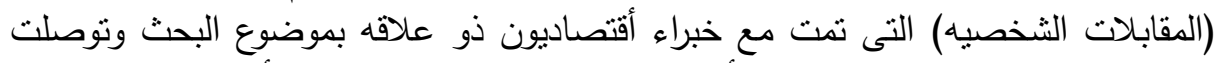

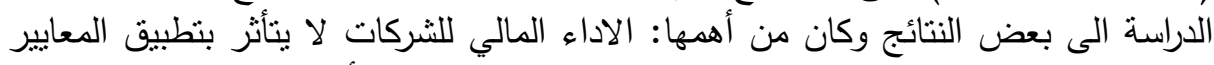

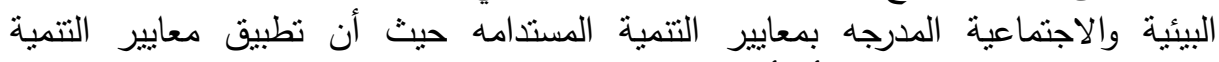

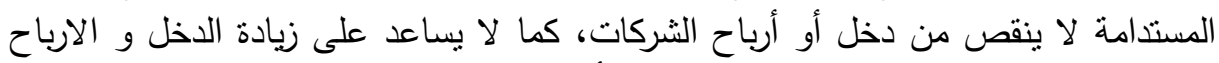

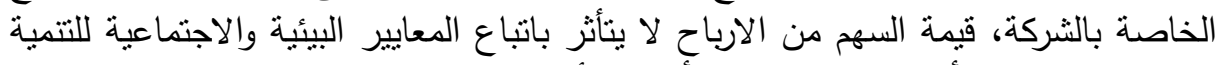

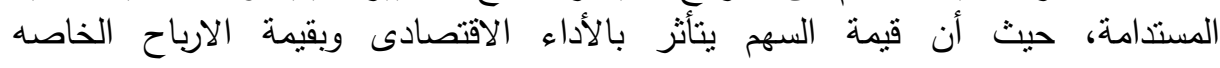

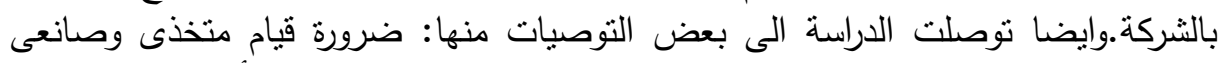

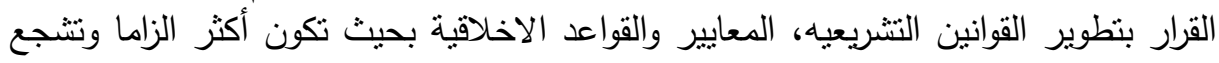

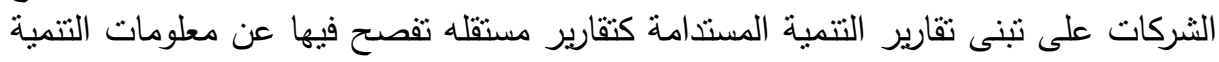

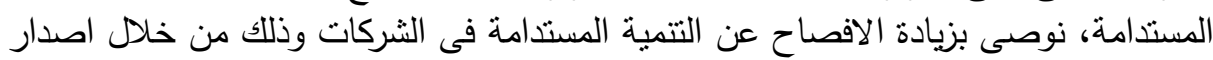
تقرير منفصل عن تقارير القوائم المالية الختامية لما لهذه التقارير من أهمية. 


\section{المُهمبهمة}

أثنادت لجنة الاستدامة الثابعة للأمم المتحدة بجهود البورصة المصرية وذلك بمؤتمر

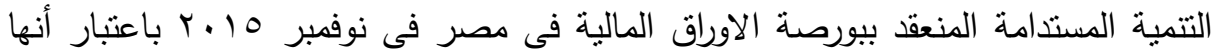
إحدى أهم الأسواق العالمية والأسواق الرائدة في مجال التتمية المستدامة؛ لكونها قد دشنت فئن مؤشر الاستامة S\&P/EGX ESG بالتعاون مع مؤسسة ستاندرد آند بورز ويأني تششين

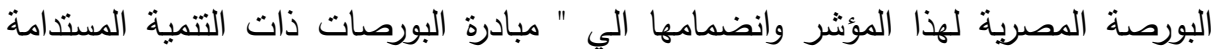
المجتمعية " Sعام 9 . . في إطار اهتمامها بالاتجاهات العالمية الحديثة في مؤشرات أسواق

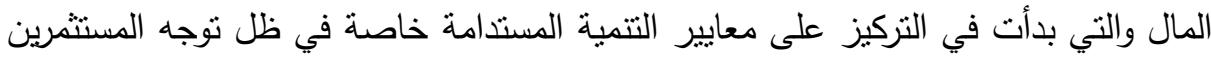
اللربط بين المعايير المالية ومعايير الأنشطة غير التقليدية للثركات، والحفاظ على مستويات أداء بيئي جيد ومسؤولية اجتماعية متميزة للثركة.وتعد مبادرة الأمم المتحدة لاستدامة أسواق التئي

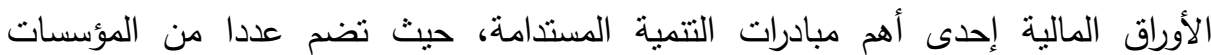

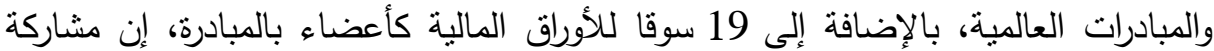
البورصة كإحدى الأسواق الرائدة في الثرق الأوسط وشمال أفريقيا في مبادرة استدامة أسواق

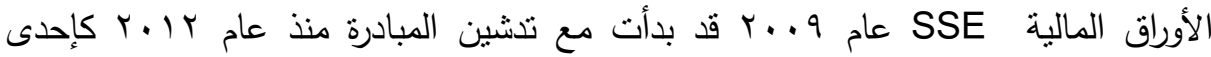

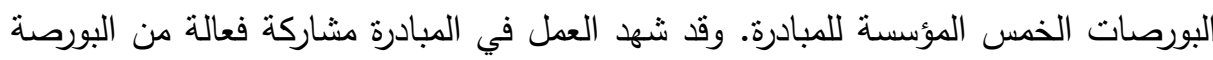

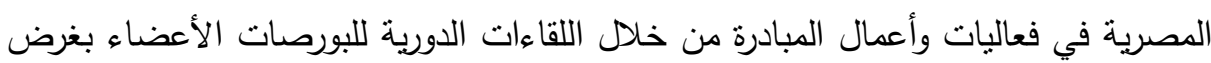
مناقثة أهم المستجدات فيما ينعلق بآليات العمل على زيادة الوعي بأهمية التتمية المستدامة العادية في سوق الأوراق المالية وتحديث أطر عمل الأسواق بما يضمن زيادة الثفافية. وقد تم تثككيل

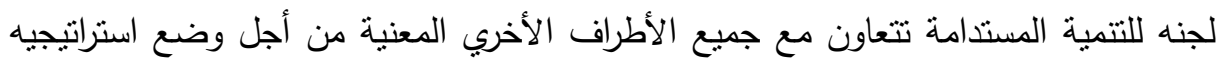

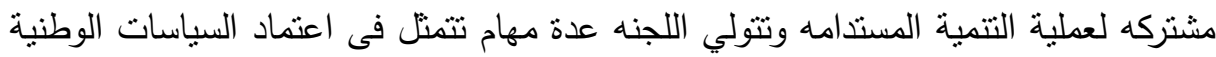

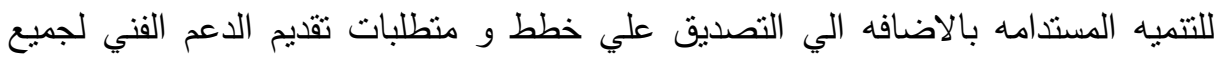
الجهات الوطنية المعنية بتحقيق التتميه الدستدامة والتقيم والتصديق علي خطط العمل والتمويل المقترحه. كما ان هناك اهتمام متزايد بدعم ونثر تطبيقات مبادئ ومعايير الدسؤولية المجتمعية بين الثركات الصصرية، حيث نتير الدراسات إلى دور المسؤولية المجتمعية في

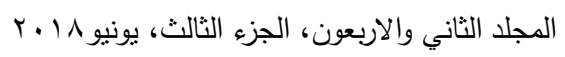


تتمية أداء الثركات وتحسن وضعها المالي، بالإضافة إلى الفائدة التي تعم على المجتمع من

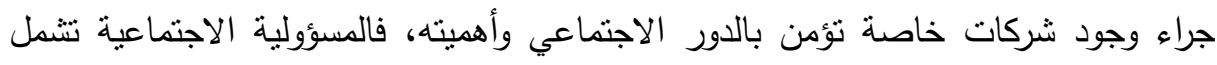
نظم العمل الداخلية وترشيد التعامل مع البيئة ومراعاة حقوق العمال ومحاربة الفساد والالتزام بالقواعد إلى جانب تحقيق العدالة بين الجنسين تحت مظلة الاستدامة

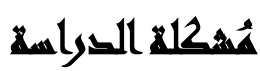

تطور مفهوم مسئولية الثركات بشكل ملحوظ فى بداية القرن الحالي حتي أصبحت تمثل

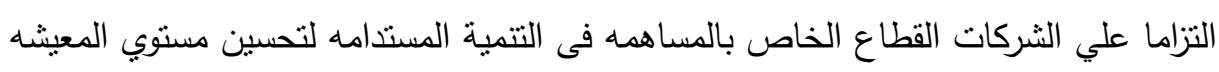
بأسلوب يحقق أهداف المساهمين ويخدم التتمية المستدامه فى ان واحد. ولم يعد تقييم الثركات

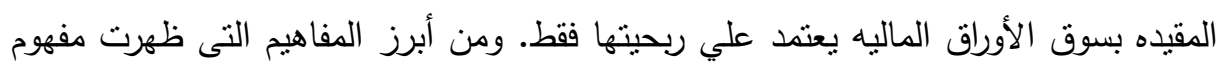
"مسئولية الثركات" حيث أصبح دور شركات القطاع الخاص محوريا فى عملية التنمية وقد

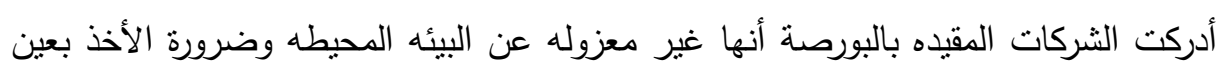
الاعتبار الأضلاع الثلاثه وهى النمو الأقتصادي والتقدم الاجتماعي وحماية البيئه ويستتد

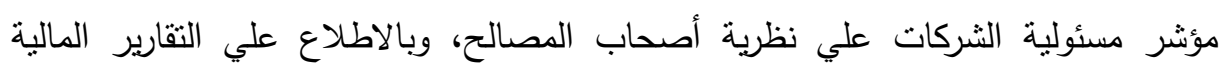

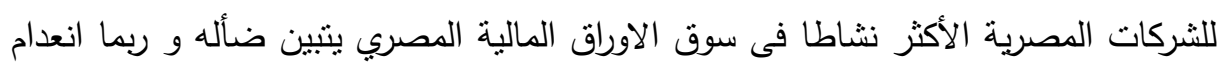

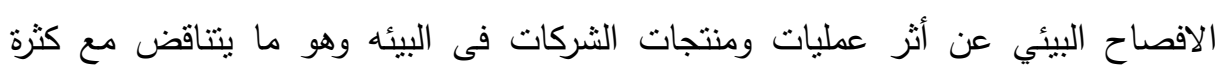
التشريعات البيئية وزيادة اهتمام المجتمع المصري بالحفاظ علي البيئه وذلك لعدم وجود معايير للتتمية المستدامه والتي تتعلق بالمسئولية البيئيه و المسئولية الاجتماعية والاداء المالي ويناء علي ذلك تتلخص مشكلة البحث فى عدم وجود موائمه بين النظرية والتطبيق فعلي صعيد النظرية تظهر مشكلة تكاليف الوكاله التي تتلخص فى سلوك الادارة وقدرتها على تحقيق

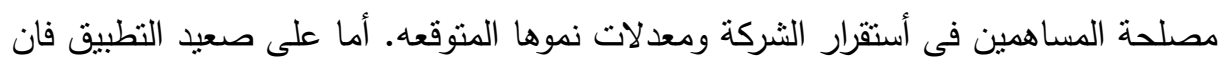
المشكلة فى معالمها تصبح أكثر نثابكا لوجود أطرافا متعدده من مستثرين فى الأوراق

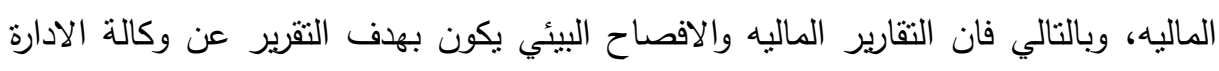

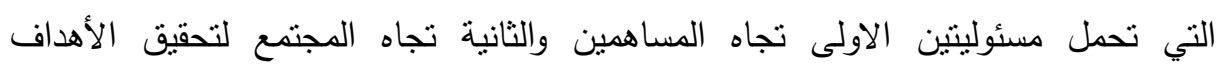

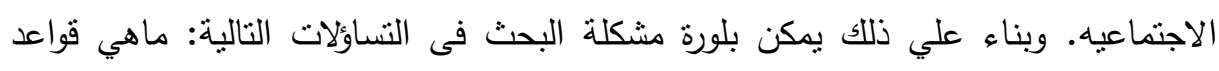

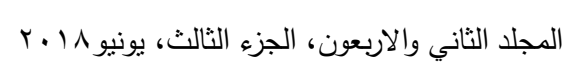


ومعايير التتمية المستدامه التي لها تأثثر هام علي الأداء المالي للمنشأه وقيمتها السوقية؟هل هناك أهمية

للالنزام بتطبيق معايير التتمية المستدامه علي جودة التقارير المالية وقرارات المستثمرين؟هل نوجد علاقة جوهرية بين معايير التتمية المستدامه والمسئولية الاجتماعية

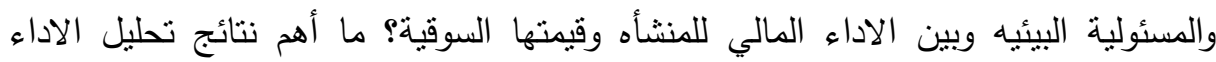
المالي والاجتماعي والبيئي للشركات المصرية محل الدراسة؟ وبناء علي ذلك يمكن بلورة مشكلة البحث فى التساؤلات التالية: 1-ماهي قواعد ومعايير التتمية المستدامه التي لها تأثنر هام علي الأداء المالي للمنشأه وقيمتها السوقية؟ ז-هل هنالك أهمية للالتزام بتطبيق معايير التتمية المستدامه علي جودة التقارير المالية وقرارات المستثمرين؟ r-هل توجد علاقة جوهرية بين معايير التنمية المستدامه والمسئولية الاجتماعية والمسئولية

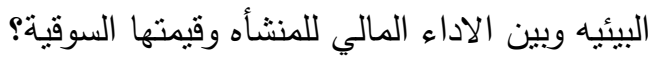
ع-ما أهم المبادرات الدولية الخاصة بالتتمية المستدامه وما أهم النظريات حول المسئولية الاجتماعية والبيئية للشركات؟

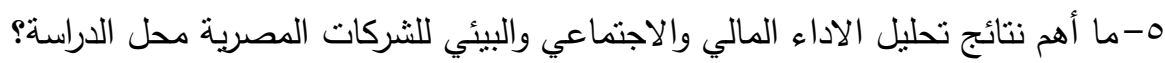
هل سيؤثر ذلك علي قرارات المستثمرين؟

\section{أهمية الصواسم}

وتأتي أهمية الدراسة الحالية من خلال ما يلي:

الأهميه النظرية: نسعى الدراسه الي ارساء الاطار النظري والمفاهيمي للتتمية المستدامه

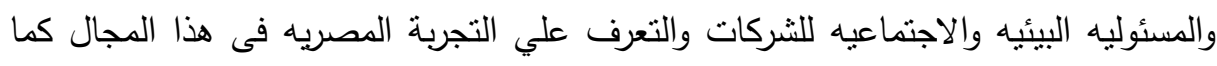
تهدف الي قياس أثر المسئوليه البيئيه والاجتماعيه علي الاداء المالي للشركات المقيده

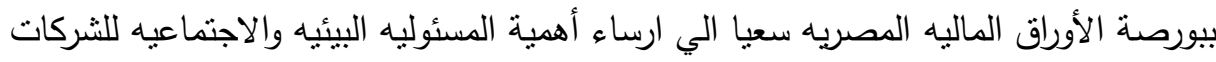

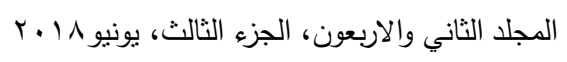


فى دعم المجتمع المصري و الارتقاء به بما يسهم فى تحقيق التتميه الأقتصاديه والاجتماعيه المستدامه.

كما نسعي الدراسة ايضا الي المساهمه فى سد الفجوه والنقص الثنديد فى الدراسات علي مستوي الاسواق المتقدمه والناشئه التي اهتمت ببحث وتقييم العلاقة بين معايير التتمية

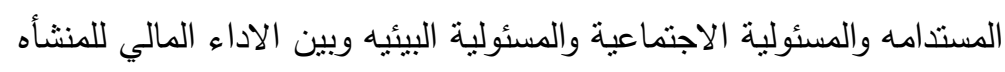

\section{الأهمبة التطبيقية:}

- يساعد هذا البحث فى امداد الجهات المسئوله عن وضع معايير التتمية المستدامه ومعايير المحاسبه فى مصر بالمعلومات التي نساعدهم فى تطوير اطار اعداد وعرض التقارير

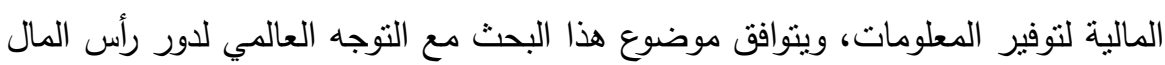
الخاص فى التتمية المستدامه.

- يساهم هذا البحث فى التعرف علي واقع الافصاح عن قواعد معايير التتمية المستدامه والمسئوليه البيئيه والأجتماعيه.

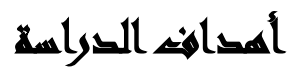

تسعى هذه الدراسة الى تحقيق مجموعة من الاهداف يمكن بلورتها فى الآتى: يتمنل

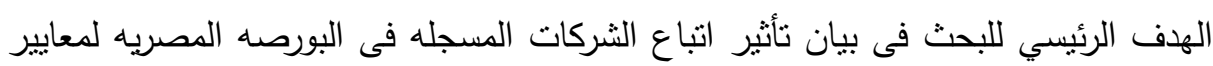

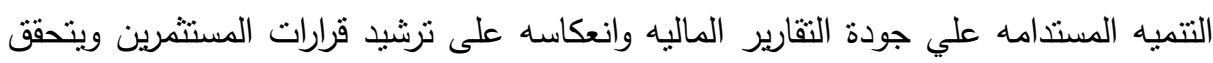
ذللك من خلال مجموعه من الأهداف الفرعيه التالية: - التعرف علي أهمية تطبيق معايير التتمية المستدامه وأثر ذلك علي شفافيه القوائم والتقارير الماليه وتعزيز ثقة المستثرين فى تقارير الرقابه بما بيساعدهم فى اتخاذ القرارات

$$
\text { الاسثماريه. }
$$

- تفعيل الرقابه علي الثركات فى محاوله لتحسين مستوي اتباع التتميه المستدامه مما يعمل

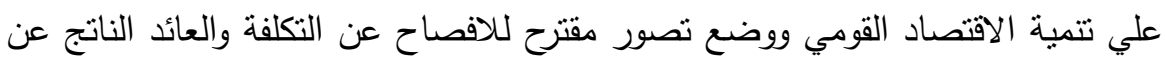

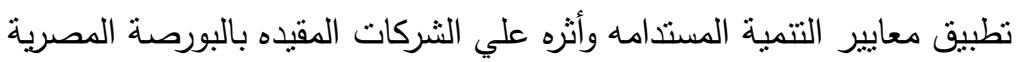

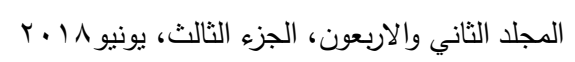


- دراسة أثر قصور معايير التتمية المستدامه فى إلزام الثركات المصرية بالافصاح عن المعلومات البيئيه الخاصه بها.

\section{هزوض القواسما}

• الفرض الأول: لا يوجد علاقة بين الاداء المالي للشركات والمسئوليه الاجتماعية المدرجه هواه بمعايير التتمية المستدامه.

• الفرض الثاني: يوجد علاقة موجبة بين حجم الثركات وبين أتباع معايير التتمية المستدامة • الفرض الثالث: لا يوجد علاقة معنويه موجبة اتباع المعايير البيئية والاجتماعية للتنمية

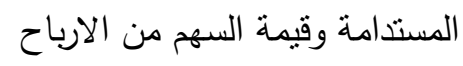

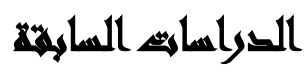

- سامتح أحمد عبد الرحيم محمد ترك (ع ا ـ Y) بعنوان "أليات الحوكمة البيئيه للمنشأت فى البيئه المصرية وانعاكسها علي قرارات المستثمرين" رسالة ماجستير، كلية

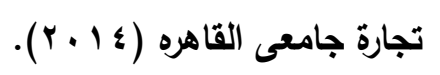

تتاولت هذه الدراسة قياس مستوي الرقابة على منثأت المراجعه فى مصر فى محاولة

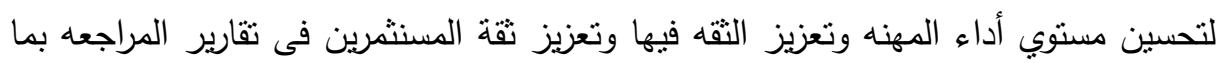
يساعدهم فى اتختنت القراران الاستثمارية. توصلت الدراسة إلى بعض النتائج والتى تتلخص فى ان حوكمة منشأت المراجعه تؤثر علي جودة عمليات المراجعه ويوجد تأثثر ايجابي لنطبيق اليات حوكمة منشأت المراجعه علي قرارات المستثرين فى سوق الاوراق المالية كما ان حوكمة منشأت المراجعه تعني فرض تئه

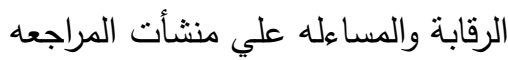


وانتهت الدراسة إلى توصيات أهمها: يجب على الثركات خاصة المقيدة بالبورصة المصرية ان تضع مؤشرات أو مقاييس خاصه بقواعد ومعايير حوكمة الثركات والمسئولية البيئية لما لهذه المؤشرات من أهمية على الثركة والمستثمرين والعاملين والبيئه.

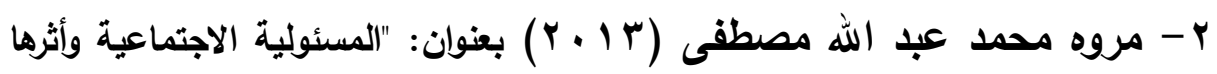

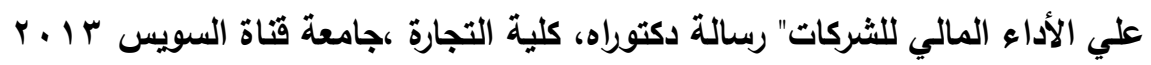

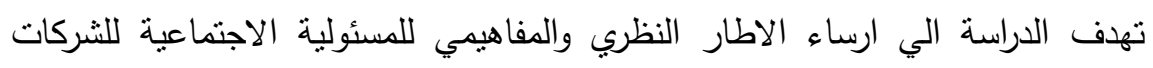

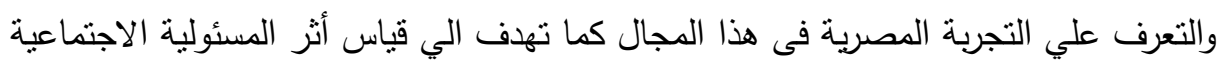
علي الاداء المالي للشركات المقيده ببورصة الأوراق المالية وانتهت الدراسة إلى نتائج أهمها:

توصلت نتائج القياس الي وجود علاقة عكسيه بين الاداء والمسئولية الاجتماعية للشركات الامر الذي قد يرجع الي حداثة تطبيق الثركات المصرية لممارسان المسئولية

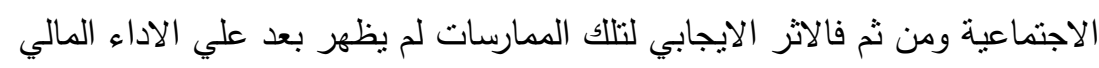

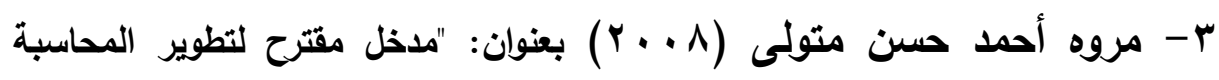
الادارية البيئية بمنظمات الاعمال لتحقيق أهداف التنمية المستدامة" (دراسة تطبيقية)

$$
\text { رسالة دكتوراه ، كلية تجارة ، جامعة القاهرة( م . . ץ). }
$$

يمكن إبراز أهم أهداف الدراسة فى الأتى: هدفت هذه الدراسة الى هلى تقييم الاتجاهات المعاصرة

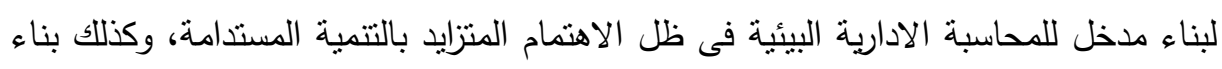

مدخل مقترح لنطوير المحاسبة الادارية البيئية.

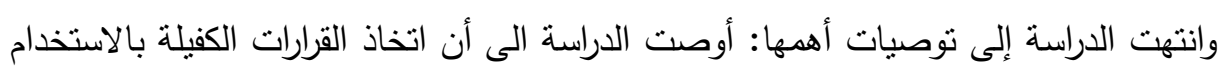

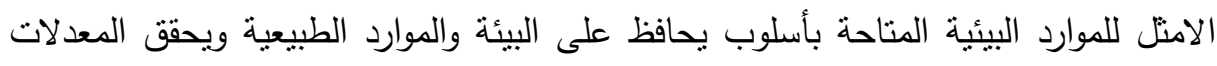

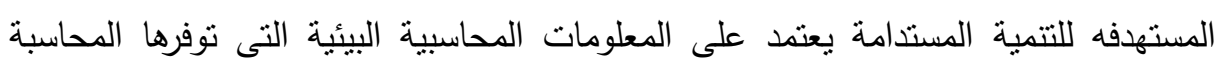
الادارية البيئية، كذللك فان المحاسبة الادارية البيئية هى مرحلة جديده من مراحل تطور المحاسبة الادارية فهى تقوم بأداء الوظائف المعتادة للمحاسبة الادارية ولكن من منظور بيئي. 
ع - بشر محمد موفق(Y . . . P) بعنوان "المحاسبة عن التمية المستدامة من منظور الثركات الانتاجية ".رسالة ماجستير، معهد الدراسات والبحوث البيئة، جامعة عين

يمكن إبراز أهم أهداف الدراسة فى الأتى: هدفت هذه الدراسة الى كيفية المساعدة فى قياس

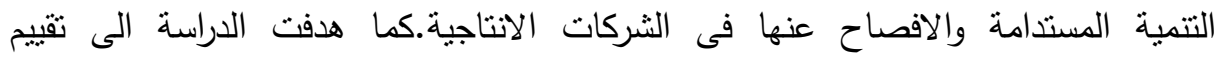
الاتجاهات المعاصرة لبناء مدخل للمحاسبة الادارية البيئية فى ظل الاهتمام المتزايد بالتتمية المستدامة، وكذلك بناء مدخل مقترح لنطوير المحاسبة

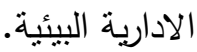

وانتهت الدراسة إلى نتائج أهمها: وتوصلت الدراسة الى نموذج مقترح لتحديد رأس المال الامثل

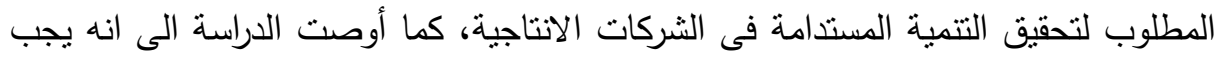
أن تهدف الثركات، بصوره رئيسية الى الحفاظ على الانتاجية، ويتطلب ذلك وجود مؤشرات كافيه، وربطها مع المؤشرات الاقتصادية مثل الانتاج (معبر عنه ماديا ونقديا). وبذلك يمكن الافصاح عن الارتباط بين النشاط الاقتصادى ولفي والنشاط البيئى ضمن تقارير البيئة التى تصدرها الثركات. كما توصلت الدراسة الى ألى أن اتخاذ القرارات الكفيلة بالاستخدام الامثل للموارد البيئية المتاحة بأسلوب يحافظ على البيئة

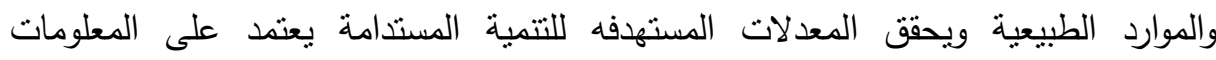
المحاسبية البيئية النى توفرها المحاسبة الادارية البيئية، كنلك فان المحاسبة الادارية البيئية هى مرحلة جديده من مراحل نظور المحاسبة الادارية فهى تقوم بأداء الوظائف المعتادة للمحاسبة الادارية ولكن من منظور بيئي. ما يضيفه موضوع الدراسة إلى الدراسات السابقة: • التعمق فى عناصر التتمية المستدامة وعدم الوقوف فقط عند الارات المحاسبة عن التتمية المستدامة من حيث تعريفها وأهدافها واطارها بصفه عامة وأهميتها فى قياس أداء المنشأه.

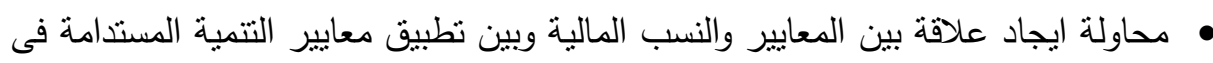
الثركات. 
محاولة حصر والوصول الى مزايا وعيوب أو التكلفه والعائد على الثركات المتبعه والتى تطبق معايير التتمية المستخامة. الاستفادة من التجارب الدول المتقدمة فى تطبيق معايير التنمية المستدامة بلأبعادها الثلاثة

\section{الإسار النظظيه}

خطة الدراسة: حيث يتتاول الباحثين في دراستهم المعايير والمؤشرات والنسب الماليه

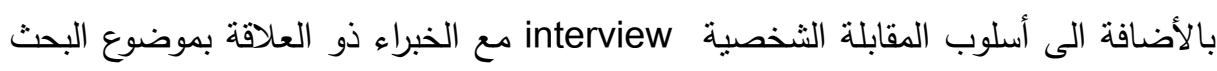
والتوصل و التعرف علي مدي تأثنير اتباع معايير التتمية المستدامة والافصاح عن الاداء

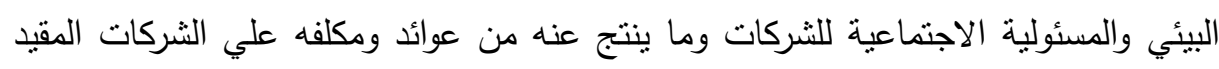
بالبورصة المصرية للاوراق الماليه. وذللك من خلال دراسة النقاط التالية: - - طبيعة وأهمية مؤشر ومعايير التتمية المستدامه والافصاح عن الاداء البيئي للمنشأه

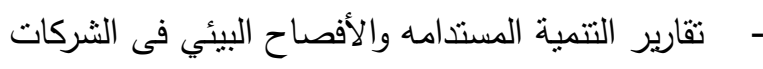

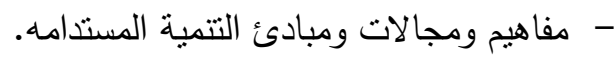
- العوامل المؤثثره علي الأداء المالي للشركات (العائد والتكلفه المؤثره علي الأداء المالي للشركات) - العلاقة بين تطبيق معايير التتمية المستدامه - أثر تطبيق معايير التتمية المستدامه و ما ينتج عنه من تكلفه وعائد على الثركات المقيده بالبورصة

\section{إجباعاهت التواسمة}

حدود الدراسة:

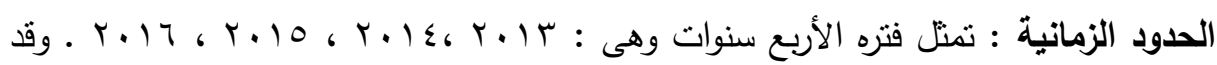

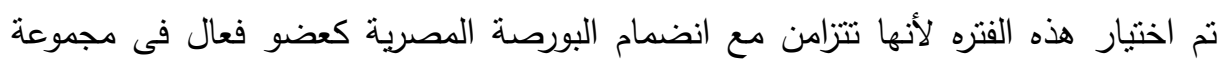

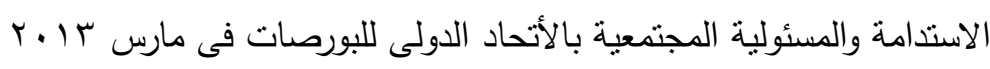

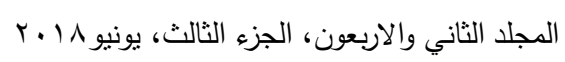


الحدود المكانية: تتمنل فى شركات مدرجة فى بورصة الاوراق المالية المصرية، اى انها تتداول بشكل أساسى فى جمهورية مصر العربية. منهج الاراسة: المنهج الوصفى: من خلال الاعتماد علي المصادر المتمنلة فى الكتب والرسائل والدوريات والمؤتمرات والندوات وغيرها سواء العربية أو الاجنبية ذات العلاقة بموضوع البحث وذلك بغرض تحليلها والاستفاده منها فى صياغة الجوانب النظرية لهذا البحث. المنهج التحليلى: وذلك من خلال استخدام المعايير والمؤشرات والنسب الماليه ذلك لعدم وجود

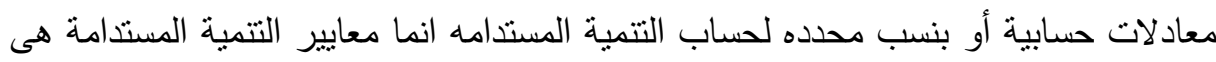

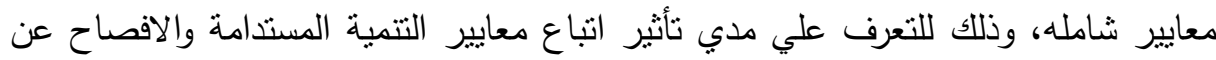
الاداء البيئي والمسئولية الاجتماعية للشركات وما ينتج عنه من عوائد ومكلفه علي الثركات المقيد بالبورصة المصرية للاوراق الماليه. المنهج الكمى: بدراسة أراء الخبراء عن طريق استخدام أسلوب interview(المقابلات الثخصيه) التى تمت مع خبراء أقتصاديون ذو علاقه بموضوع البحث ذللك للحصول علي بلي

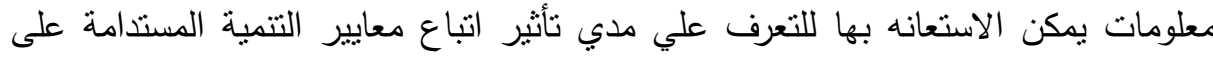
الاداء البيئي والمسئولية الاجتماعية للشركات

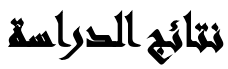

حرصت الحكومة المصرية على دعم بيئة الاستثمار فى البورصة المصرية و كان من أهم الاجراءات التى اتخذتها لتحقيق هذا الهدف هو اصدار قانون سوق رأس المال رقمه لسنة r991 واللائحه التتفيذية له والذى يعتبر حجر الاساس فى تتشيط التعاملات فى الهى البورصة المصرية خلال السنوات التى تلت تطبيقه وذلك لادخال معايير التتمية المستدامة المناسبة والحوكمه لتطبيقها بالثركات فى السوق المصرية 
نتائج الاراسة العملية: يمكن أن نستخدم ونعتمد على النسب والمؤشرات الماليه كمقياس

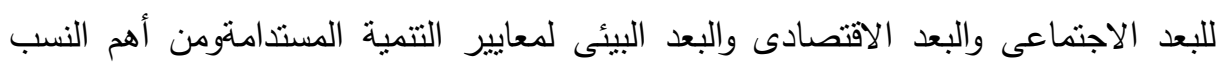
المالية التى تم استخدامها

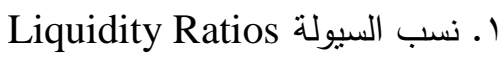

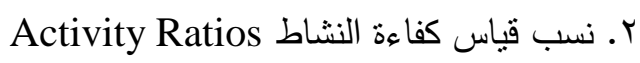

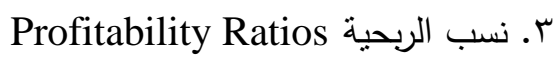

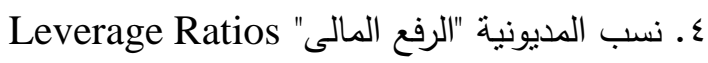

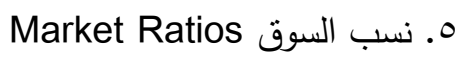

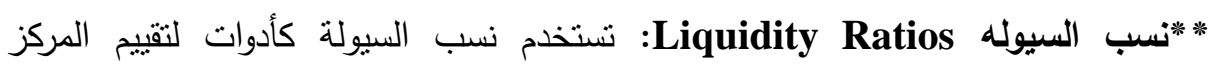
الأثتماني للمنشأة والذي يعبر عادة عن مدى قدرتها في الوفاء بالتزاماتها قصيرة الأجل ، ومن لهن

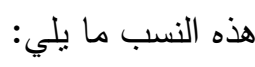
: Current Ratio نسب التداول نسب التداول= $\quad$ نسب

: Quick Acid Ratio نسبة السيولة السريعة *

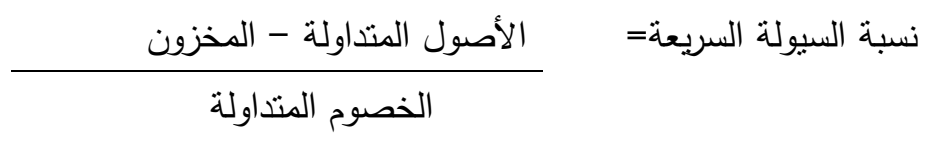
والناتج يعتبر مؤشر لكفاءة الإدارة **نسب النشاط Activity Ratios: نستخدم هذه النسب لنقييم مدى نجاح إدارة المنشأة في

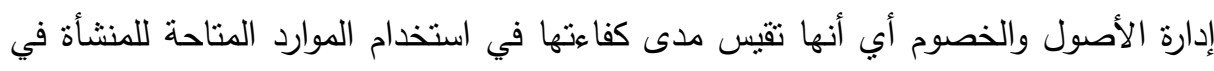

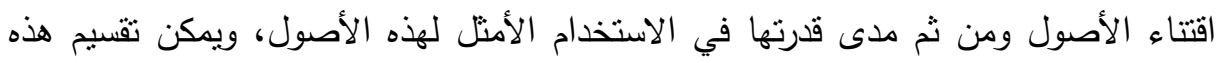

$$
\text { النسب أو المعدلات في مجموعنين رئيسيتين هما: }
$$
1-نسب أو معدلات دوران الأصول والخصوم المتداولة وتتشل ما يلي: 


$$
\text { * معدل دوران المدينين = متوسط المدينين }
$$

ويقيس هذان المعدلان كفاءة إدارة الأتثمان ومدى فاعلية سياسات الائمان والتحصيل

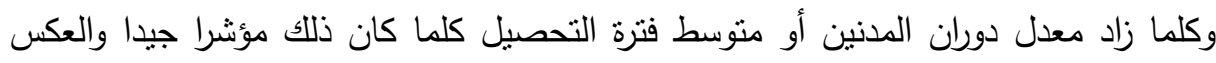

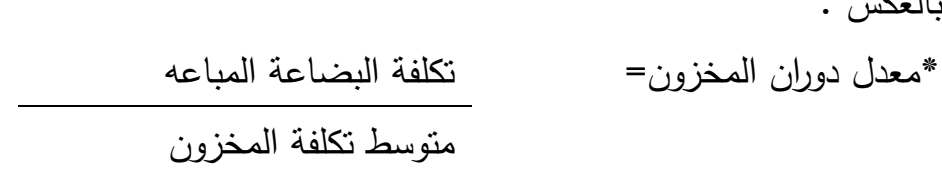

ويقيس هذان المعدلان مدى كفاءة وفاعلية إدارة المخزون وكلما زاد معدل دوران

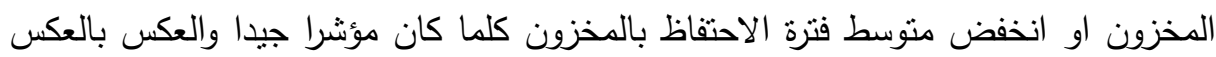

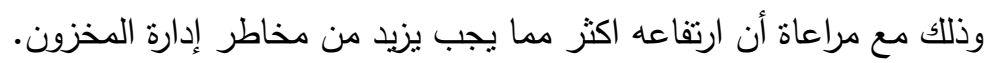

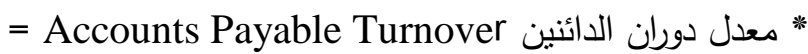

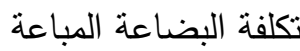
رصيد الدائنين

ويقيس هذان المعدلان مدى نجاح تحقيق الملاءمه بين سياستي البيع والثراء ، لذا كلما

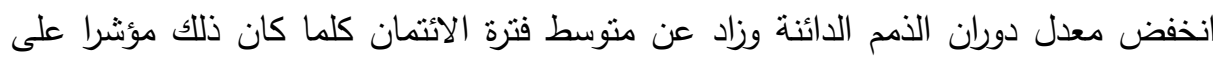
تخفيض الضغوطات التي ستواجهها المنشأة من زاوية السيولة، ذلك سيزيد من طول الفترة

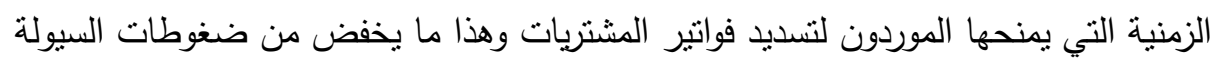

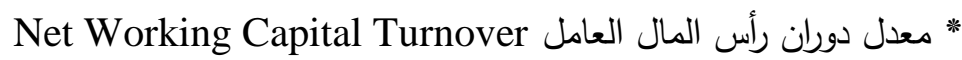

$$
\text { صافي المبيعات }
$$

وكلما زاد هذا المعدل كان دليلا على كفاءة إدارة صافي رأس المال والعكس صحيح.

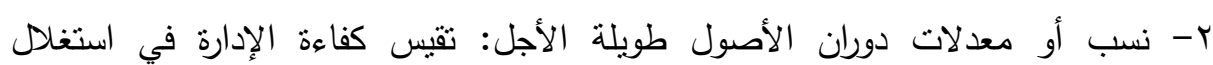

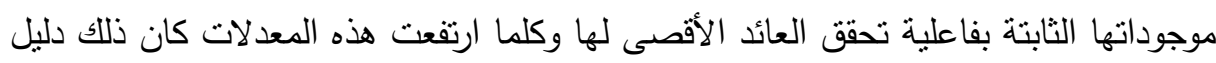

$$
\text { جودة والعكس بالعكس، ومن أهم المعدلات: }
$$

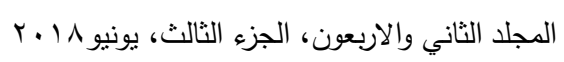


Total Assets Turnover معدل دوران الأصول *

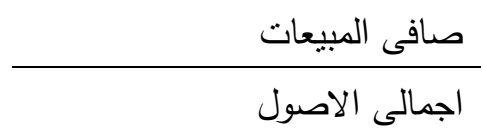

وكما نرى فإن هذه المجموعة تهنم كثثراً بمعدلات الدوران ، ومتوسط فترات الاداء التشغيلى كفاءة إدارة أصول الثركة.

**نسب الريحيه Profitability Ratios: وتهنم نسب الربحية بقياس قدرة الثركة على توليد الارباح من انشطها التشغيلية ، ويفضل تقسيم نسب الربحية الى نوعين على النحو أ - النسب التى تقيس ربحية الثركة استتاد إلى مبيعات الثركة . ب - النسب التى تقيس ربحية الثركة استتاد الى حجم الاستثمارات فى الثركة . وكذلك ايضاً يمكن تتسيب صافى الربح لاى من حسابات وبنود القوائم المالية ، للوقوف لفائ على علاقة مساهمة هذا البند فى تحقيق الربح

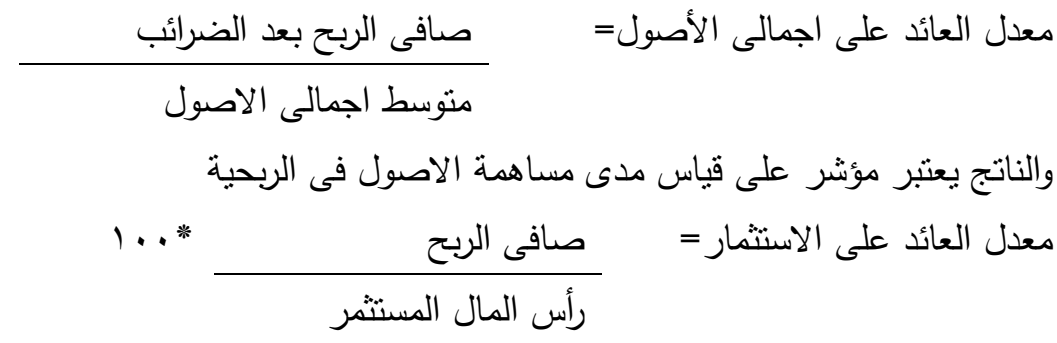

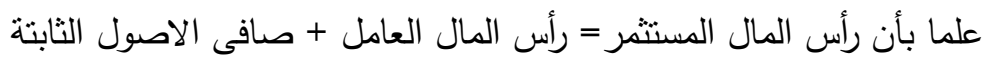
والناتج يعتبر مؤشر لقياس العائد على اجمالى الاستثمار ران

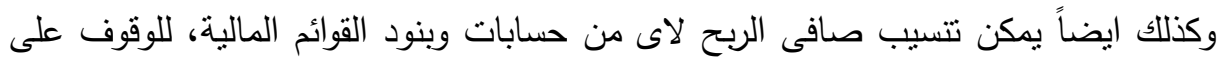
علاقة مساهمة هذا البند في تحقيق الربح. نسب السوق Market Ratios: مجموعة نسب ذات الهية خاصة لحملة الاسهر، وللمستثمرين المحتملين فى الاسهم، وتضطلع هذه النسب بقياس تأثثر اداء الثركة على اسعار الاسهم العادية فى السوق استتاد الى الهدف العام للادارة التمويلية وهو تعظيم ثروة المساهمين عن طريق تعظيم السوقية للسهم. 


$$
\begin{aligned}
& \text { نصيب السهم من الربح= مافى الربح بعد الضرائب - توزيعات الاسهم الممتازه }
\end{aligned}
$$

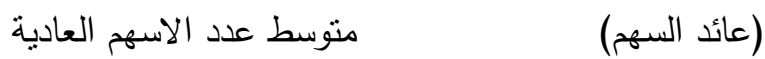

$$
\begin{aligned}
& \text { نسبة توزيعات الارباح= نصيب السهم من التوزيعات } \\
& \text { نصيب السهم من الارباح } \\
& \text { ريع السهم = حصة السهم من الارباح الموزعة } \\
& \text { القيمة السوقية له }
\end{aligned}
$$

مما سبق نستخلص نتائج مدى اتباع وتطبيق معايير التنمية المستدامة بقطاع أناع البتروكيماويات: حيث يتم اعطاء رقم ا فى حال اتباع أى من معايير التتمية المستدامة،

\begin{tabular}{|c|c|c|c|c|}
\hline النـاتـــــ & الاقتصاديية & الاجتماعيية & البيائييّة & الشركـــــــة \\
\hline$\mu$ & 1 & 1 & 1 & شركة سيدى كرير \\
\hline$Y$ & • & 1 & 1 & شركة أموك \\
\hline 1 & 1 & $\cdot$ & $\cdot$ & شركة غاز مص \\
\hline
\end{tabular}

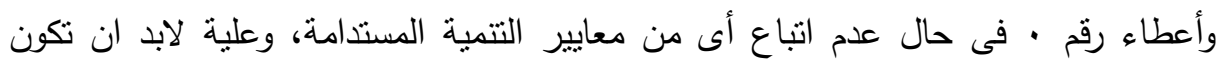

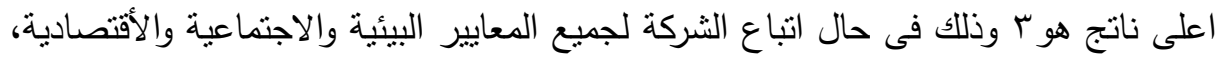
وأقل ناتج هو · وذللك فى حال انباع عدم الثركة لأى من معايير التتمية المستدامة

\begin{tabular}{|c|c|c|c|c|}
\hline النـاتــــــج & الاقتصائيية & الاجتمعاييزية & المعاييز البيئية & الشركـــــــة \\
\hline$\overline{11}$ & . & 1 & . & شركة ايكمي \\
\hline$T$ & 1 & . & . & شركة ايبكو \\
\hline 1 & . & $T$ & . & شركة النيل للَّدوية \\
\hline
\end{tabular}

كذلك نستخلص نتائج مدى اتباع وتطبيق معايير التمية المستدامة بقطاع الصناعات

الدوائية

وعليه يتضح أن الثركات بقطاع الصناعات الدوائية لا يهتم باتباع تطبيق معايير التنمية المستدامة بمجالاتها البيئية والاجتماعية والاقتصادية. 
خالد حسين أحمد أبو حسين وآخرون

ونستخلص نتائج مدى اتباع وتطبيق معايير التتمية المستدامة بقطاع الصناعات

الكيماوية:

\begin{tabular}{|c|c|c|c|c|}
\hline النـاتـــــج & الاقتصعاديية & الاجتماعيية & البيئية المعيز & الشركـــــــة \\
\hline r & - & 1 & 1 & شركة مصر لصناعة \\
\hline . & . & . & . & شركة سماد مصر \\
\hline 1 & 1 & . & . & ثُركة المالية والصناعية \\
\hline
\end{tabular}

وعليه يتضح أن الثركات بقطاع الصناعات الكيماويه لا يهتم بأتباع تطبيق معايير

التتمية المستدامة بمجالاتها البيئية و الاجتماعية والاقتصادية.

- ان معايير الاداء المالى للثركات تعطى فكرة للكثف عن مواضيع الضعف والقوه وبيان

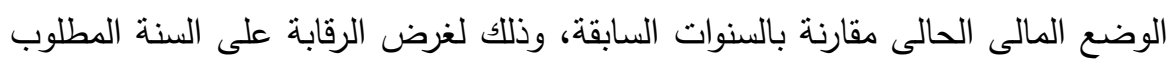

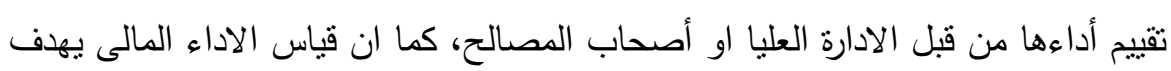
الى تحقيق القيمة لحملة الاسهم من خلال تحقيق عدد من الاهداف المالية المتمثلك فى الحي الربحيه وتحقيق معدلات نمو مرتقعه وتحسين القيمة الاقتصادية.

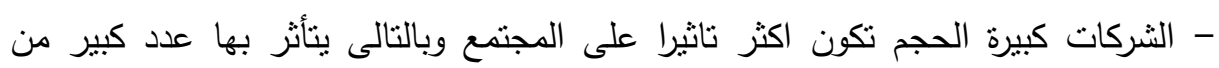
أصحاب المصالح ومن ثم تكون أكثر رغبة فى الافصاح عن التتمية المستدامة مقارنة بالثركات صغيره الحجم، وكذلك الشركات التى تحقق نمو فى ايرادات السهم، ايضا الثركات ذات الرافعه المالية الكبيره تفصح بشكل أكثر عن التتمية المستدامة . - الاداء المالي للشركات لا يتأثر بتطبيق المعايير البيئية والاجتماعية المدرجه بمعايير التتمية

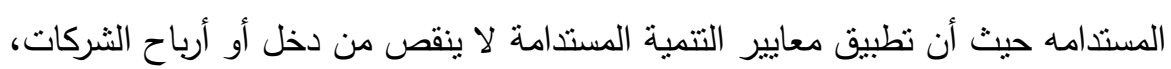

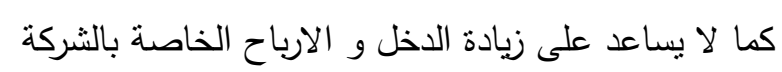

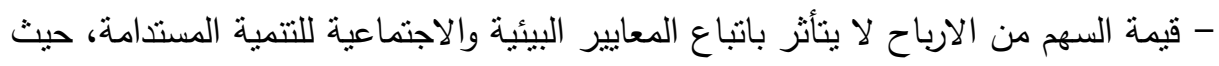

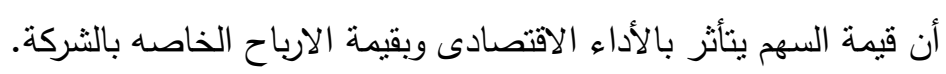
- قطاعات الأعمال التابعه لها الثركة تؤثز بدرجة كبيرة فى أتباع معايير التتمية المستدامة،

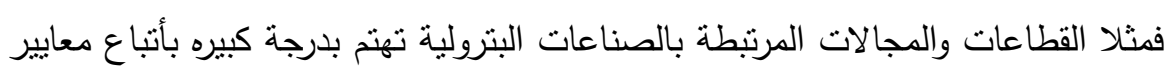

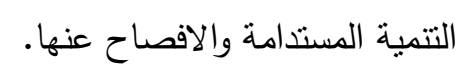

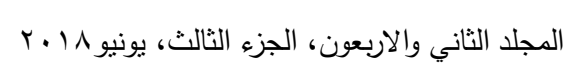


- تبين من نتائج الدراسة ان الثركات تفصح عن التتمية المستدامة فى تقاريرها السنوية وليس

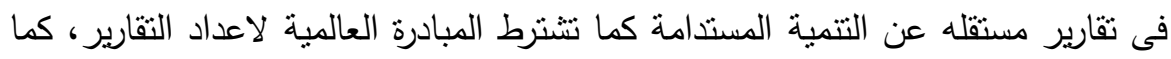
ان بنود الافصاح عن التتمية المستدامة شملت الافصاح عن صحة المجتمع، الافصاح

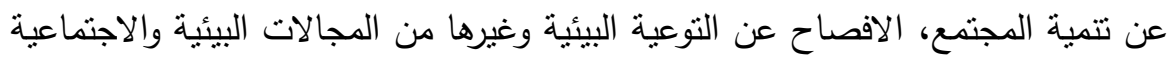
والاقتصادية.

- تين من نتائج الدراسة أن الافصاح عن التتمية المستدامة بالثركات غالبا افصاح وصفيا اكثر منه كميا.

- لا يزال هناك نقصا كبيرا فى ايجاد الصيغ المحاسبية التى تربط بين الابعاد الثلاثة للتتمية

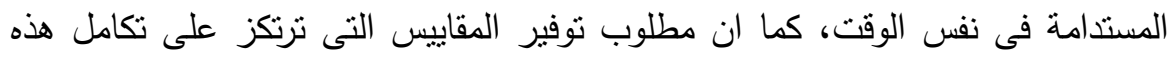
الابعاد، واثتنقاق مقاييس تتعلق براس المال الاجتماعى.

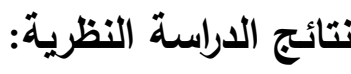
- يوجد نتشابة و اتساق للاطار العام والتفصيلى لمعايير التتمية المستدامة والمطبقة بالثركات المقيدة فى البورصة المصرية مقارنة بالاطار العام والتقصيلى لمعايير التتمية المستدامة المتعارف عليها دوليا. فى حين وجود اختلاف فى مدى وشكل الافصاح عن التتمية المستدامة بين الدول وذلك بسبب اختلاف الثقافات بين الدول، وكذلك اختلاف النظم

$$
\text { السياسية والمالية والأقتصاديه. }
$$

- يوجد تباين فى مستوى التزام الثركات فى الدول المثقدمة بمنطلبات المبادرة العالمية لاعداد

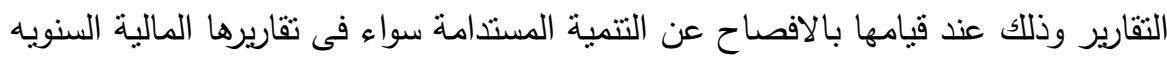

$$
\text { او تقارير الاستدامة . }
$$

- ان التتمية المستدامة تعتبر من المفاهيم التى نالت كثثرا من الاهتمام سواء من الناحية

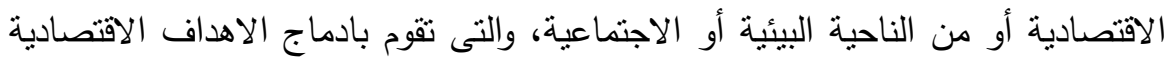

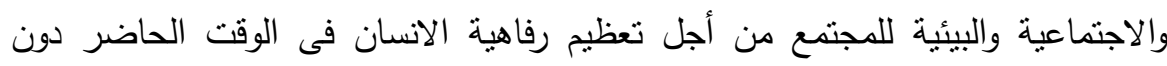
المساس بقدرة الأجيال المقبله على تلبية احتاجاتها. 
- ان أبعاد التتمية المستدامة هى ثلاثة أبعاد رئيسية تتحصر فى البيئة والاقتصاد والمجتمع، وان فاعلية التتمية المستدامة تعتمد على تضافر الجهود فى الثلاثة مجالات (المجالات التات

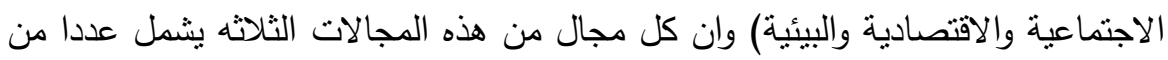
الأنشطة التى تتداخل مع بعضها بما يحقق التتمية المستدامة فى الثركات.

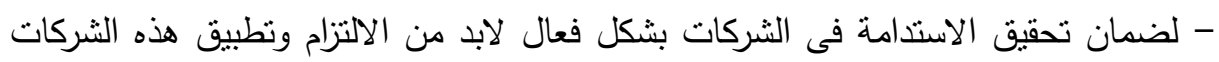
لمبادئ التتمية المستدامة والتى تتكون من ستة مبادئ خاصة لإنئ بالتتمية المستدامة.

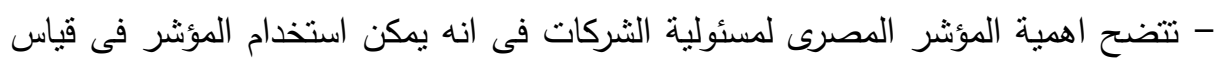

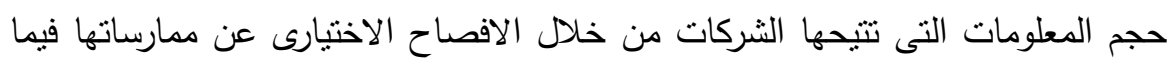
يخص الحوكمة والبيئة والمسئولية الاجتماعية، كما أن المؤشر يعتبر ضمان التهات ألا يكون قيام

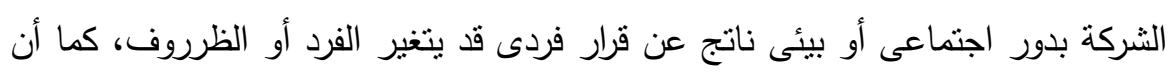

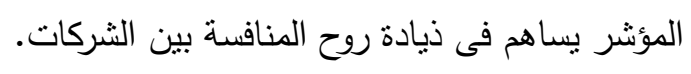

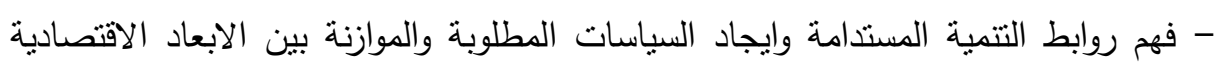

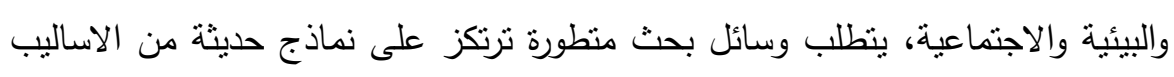

الاحصائية.

\section{المهوبياهن}

فى ضوء الدراسة النظرية والعلمية يمكن تحديد أهم التوصيات على النحو التالى:

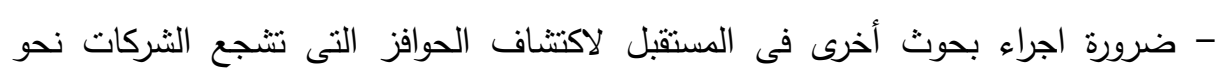
الأفصاح عن التتمية المستدامة، وبالمنل اكتشاف قيود ومعوقات الافصاح عن التتمية المستدامة داخل الثركات المصرية فى قطاعات أخرى بخلاف القطاعات التى شملتها الدراسة الحالية.

- ضرورة اجراء بحوث أخرى فى المستقبل لدراسة العلاقه بين محددات اخرى بخلاف تلك الته

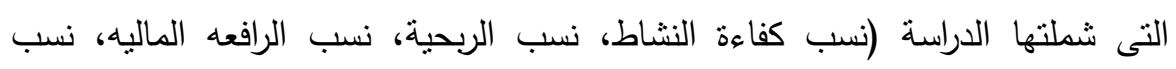
السيولة، ايرادات السهم الواحد) وبين قيام الشركات بالافصاح عن التتمية المستدامة. 
- ضرورة قيام متخذى وصانعى القرار بتطوير القوانين التشريعيه، المعايير والقواعد الاخلاقية بحيث تكون أكثر الزاما ونتجع الثركات على تنبى تقارير التتمية المستدامة كتقارير

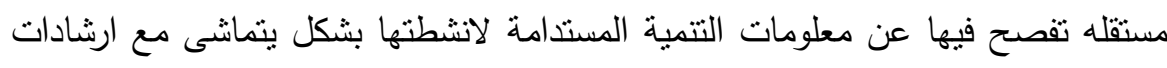
المبادرة العالمية لاعداد التقارير • - نوصى بزيادة الافصاح عن التتمية المستدامة فى الثركات وذللك من خلال اصدار تقرير

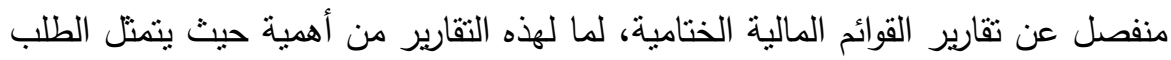
على تقارير التتمية المستدامة فى حاجة أصحاب المصالح (الداخلين والخارجين) للمعلومات عن الأداء الاجتماعى والاقتصادى والبيئى للشركات.

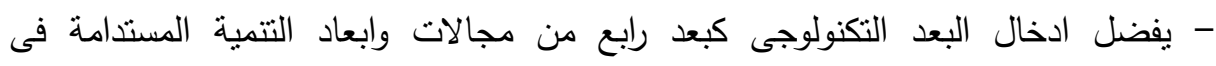

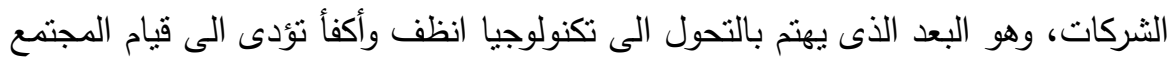
بأستخدام أقل قدر من الطاقة والموارد، ولكى تصبح التكنولوجيا فى صالح الاقتصاد والمجتمع والبيئة وتؤدى فى ذات الوقت الى أفضل تتمية مستدامة فى الثركات.

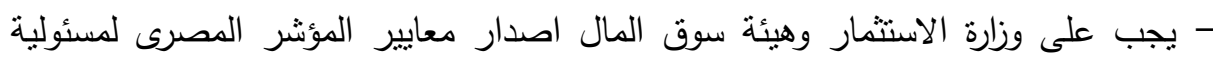

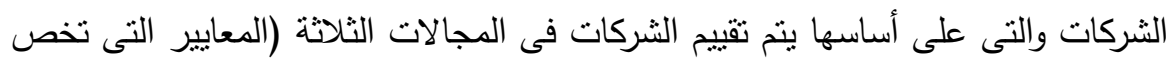

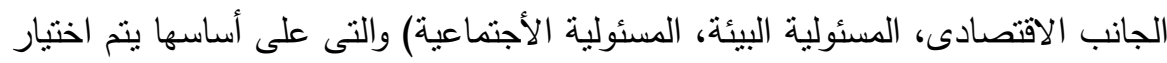

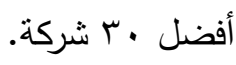
- يجب على الشركات المسجلة بالبورصة المصرية - خاصة شركات المؤشر المصرى- أن تضع مؤشرات أو مقاييس خاصة بقواعد ومعايير حوكمة الشركات والمسئولية الاجتماعية والمسئولية البيئية والجوانب الاقتصادية، لما لهذه المؤشرات من أهمية على الثركات والمستثمرين والعاملين والبيئة. - ضرورة الزام الثركات باعتماد الاستدامة الخاصة بها من مراجع خارجى منلما هو الحال فى التقارير المالية السنوية. 
- ضرورة قيام هيئة الرقابة المالية باصدار لائحه جديدة حول القواعد التنفيذيه لحوكمة

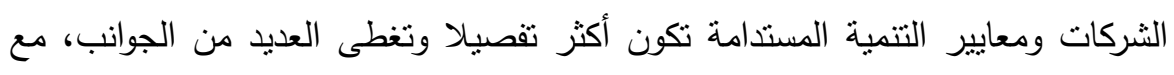

$$
\text { وضع جدول زمنى مندرج لالزام الشركات بنطبيق نلك البنود. }
$$

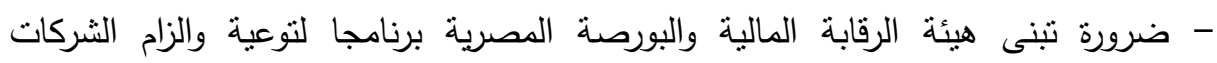
والمستثمرين بأهمية تطبيق معايير التتمية المستدامة.

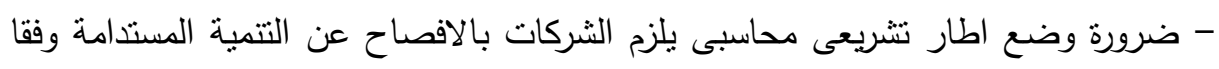

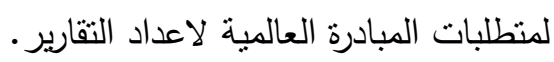

- يفضل الفصل بين النفقات البيئية والتكاليف التشغلية بصورة عامة، عند نوزيعها على لـانى العمليات والمنتجات أو الموازنات من قبل الثركات.

- عرض حجم الانفاق البيئى من قبل الثركات بصورة منفصله، بحيث يبين مدى مساهمة هذه الثركات فى حماية البيئة. - يجب أن تهدف الثركات وبصورة رئيسية للحفاظ على الانتاجية وبنفس الوقت تقليل الضرر البيئي المرافق للعملية الانتاجية.

\section{المراجي}

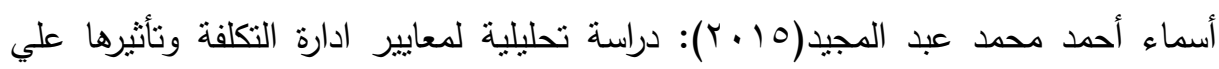

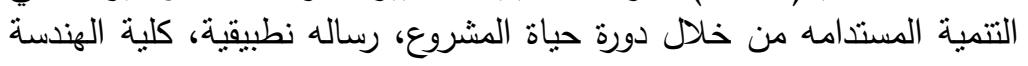
جامعة القاهره

سامح أحمد عبد الرحيم محمد ترك(ى الـ ب): دراسة امكانية تطبيق أليات الحوكمة البيئيه

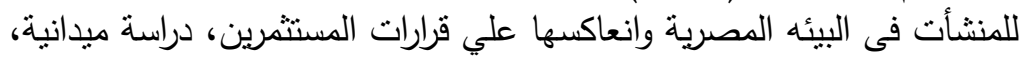
كلية التجارة جامعة القاهرة البئه الثرية

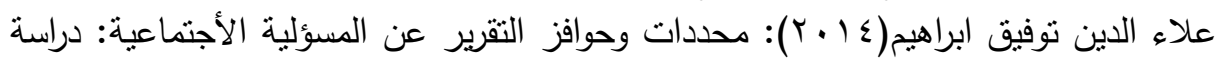

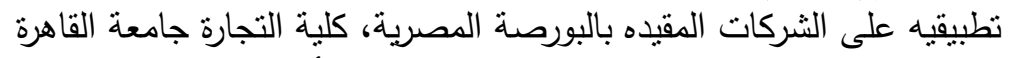

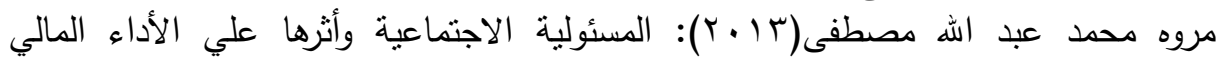

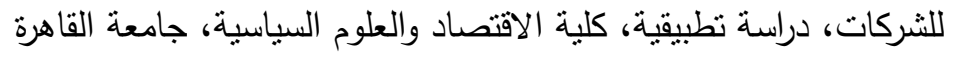


شيماء سعيد محمد يس(r ( ب): تأثثر الافصاح عن الأداء البيئي للمنشأه علي جودة التقارير

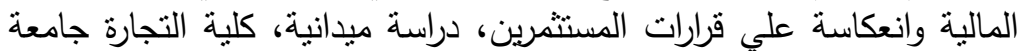
القاهزة

يونس حسن عقل، جمال علي محمد: قياس مستوي ادراك المستثمرين للمحتوي المعلوماتي

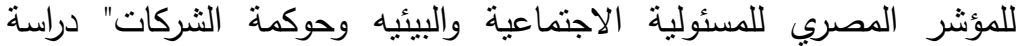

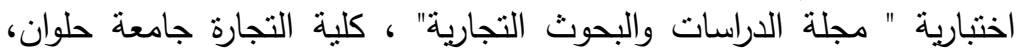

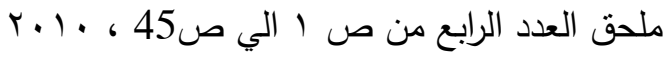
بشر محمد موفق: المحاسبة عن التتمية المستدامة من منظور الثركات الانتاجية، بحث مقدم

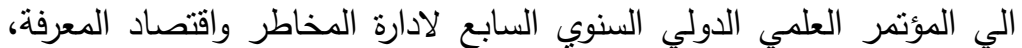
r...v

مروه احمد حسن متولي: مدخل مقترح لنطوير المحاسبة الادارية البيئيه بمنظمات الاعمال لنخقيق أهداف التتمية المستدامة دراسة نظرية تطبيقية، مجلة البحوث النية المالية

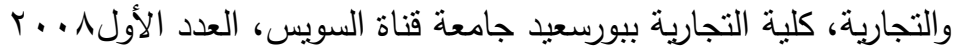
عفاف مبارك محمد: مراجعة وقياس التقرير عن التتمية المستدامه فى اطار المسأله

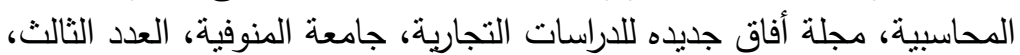

$$
\text { r... }
$$

عاطف محمد احمد أحمد: تحليل محتوي الافصاح المحاسبي عن التتمية المستدامة للشركات

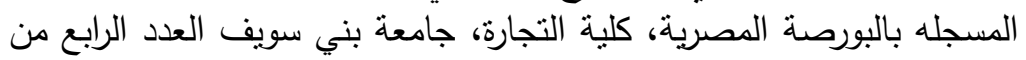
صفحه :

أديب قاسم سمشندي: الأسواق المالية وأثرها فى التتمية الأقتصادية للأوراق المالية، مجلة كلية

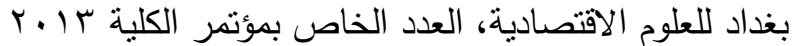

Ramanauskiene Jolite, The concept of sustainable development and its use for sustainability scenarios, Kaunas University of Technology, September 2015

Renzo Mori, sustainability reporting and assurance: a historical analysis on a world-wide phenomenon, 2014

Karthik Balakrishnan, Mandatory financial reporting and voluntary disclosure : evidence from mandatory, university of Pennsylvania, 2012

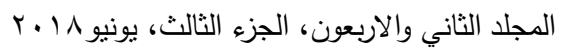


خالد حسين أحمد أبو حسين وآخرون

D.Heledd Jenkins and Louise Obara, Corporate social responsibility (CSR) in the mining industry- the risk of community dependency, Cardiff University, 2006-

D.Heledd Jenkins and Louise Obara, Corporate social responsibility (CSR) in the mining industry- the risk of community dependency, Cardiff University, 2006

Jonathan M.Harris, sustainability and sustainable development, International Society for Ecological Economics, February 2003

Thomas M Parris, what is sustainable development?, Environment Science and Policy, 2005

\title{
THE REVENUE AND THE COST FOR APPLYING STANDARDS OF SUSTAINABLE DEVELOPMENT AND THEIR IMPACT ON LISTED COMPANIES IN EGYPTIAN STOCK MARKET
}

\author{
Ahmed, Kh. H. ${ }^{(1)}$; Lotfy, M. A. ${ }^{(1)}$ and Farrag, Naglaa, A. \\ 1) Faculty of Commerce, Ain Shams University
}

\begin{abstract}
The aim of this study is to investigate the effect of the follow-up of companies registered in the Egyptian Stock Exchange on sustainable development standards on the quality of financial reports and their reflection on the rationalization of investor decisions, And to recognize the importance of implementing sustainable development criteria and the impact on the transparency of financial statements and reports and enhance the confidence of investors in the control reports to help them in making investment decisions, Two tools were used in the study: the first is to use the criteria and financial ratios because there are no accounting equations or specific percentages for the sustainable

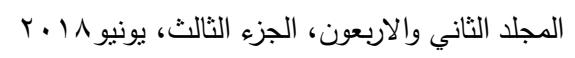


development account. However, the criteria for sustainable development are comprehensive, The second tool is the use of the interpersonal interview

method with relevant economists, The study found some results: The financial performance of companies is not affected by the application of environmental and social criteria included in sustainable development standards. The application of sustainable development standards does not reduce the income or profits of companies, The value of the share is not affected by the environmental and social criteria for sustainable development, as the value of the stock is affected by the economic performance and the profit value of the company, The study also reached some recommendations, including the need for decision-makers and legislators to develop legislative laws, ethical standards and rules so that they are more binding and encourage companies to adopt sustainable development reports as independent reports to disclose sustainable development information. During the issuance of a separate report on the reports of the final financial statements of the importance of these reports 